\title{
Analysis of Link Adaptation Algorithms Using Coding Scheme for OFDM Systems Under Frequency Selective Fading Environment
}

\author{
Sandeep Singh and Neetu Sood
}

\begin{abstract}
Orthogonal frequency division multiplexing (OFDM) is a robust technique utilized in wireless communication systems affected by frequency selectivity. In combination with multiple antennas at the transmitter and receiver as well as adaptive loading algorithms, OFDM proves to be robust against frequency selectivity. This paper presents OFDM with link adaptation algorithms applied to Multi-Input Multi-output (MIMO) systems. In this paper we apply an optimization discrete bit loading algorithm and power allocation for each subcarrier assuming instantaneous channel state information is known at both the transmitter and receiver. To improve the BER performance of wireless systems, coding techniques such as gray code and trellis coded modulation (TCM) are employed at the transmitter.
\end{abstract}

Index Terms-OFDM, SISO, MIMO, M-PSK, TCM adaptive loading.

\section{INTRODUCTION}

Demands for wireless communications are tremendously increasing. However, these desirers cannot be accomplished due to the limited frequency resources available for wireless communications. However, as the symbol duration reduces with the increase of data-rate, the systems using single carrier modulation, afflicted by more severe inter-symbol interference (ISI) caused by the dispersive fading of wireless channels, accordingly needing more complex equalization. OFDM technique divides the entire frequency selective fading channel into a number of narrow band flat fading sub-channels in which high-bit-rate data are transmitted in parallel and do not experience ISI because of long symbol duration [1], [2]. In OFDM system, channel fading on different sub-channel is different when all OFDM sub-carriers employ the same fixed modulation scheme. The error probability is dominated by the sub-carrier with the highest attenuation [3]. This problem can be overwhelmed by applying some adaptive loading algorithms.

The basic concept of adaptive loading algorithms is to adapt the wireless link efficiently in the actual channel conditions by altering a number of communication parameters.

Transmission power, symbol rate and constellation size can be dynamically adapted in response to the time-varying channel. With selection of these transmission parameters, the system makes the most out of a time varying channel, instead

Manuscript received October 12, 2012; revised November 22, 2012.

The authors are with the Dr. B R Ambedkar National Institute of Technology (e-mail: sandeep.ece04@gmail.com, soodn@nitj.ac.in) of fixing the parameters for a worst-case channel [4]. The trade off involves minimizing the error probability for robustness and enhancing the performance of the system for bandwidth efficiency. OFDM can also be combined with multi-input multi-output (MIMO) technique to increase diversity gain and achieve throughput. MIMO systems, employs multiple antennas at transmitter and receiver for data transmission and reception. The technique is termed as spatial multiplexing. Combining OFDM, MIMO and adaptive loading algorithms, all three dimension of signal transmission (time, frequency, and space) are efficiently utilized, leading to a robust, dynamic, high performance system. This paper is organized as follows: Section 2 introduce OFDM system model, Section 3 introduces the concept of Adaptive modulation, then we consider bit loading and power allocation algorithms for MIMO/OFDM system in section 4 . Section 5 shows simulation results and section 6 has conclusions.

\section{OFDM SYSTEM MODEL}

The OFDM system model in this paper has the block structure as shown in Fig. 1. The system converts the input bits stream into a number of parallel streams and maps them into complex numbers in modulator block which determines constellation points of each subcarrier based on the instantaneous channel state information. The modulator generates data symbols which are passed to IFFT block for providing orthogonality among each subcarrier. The inverse FFT at transmitter and FFT at the receiver perform reversible linear mapping between ' $\mathrm{N}$ ' complex data symbols and ' $\mathrm{N}$ ' complex OFDM symbols.

Cyclic extension is inserted to each of resulting symbols. The number of bits assigned to each subcarrier depends on the instantaneous signal to noise ratio across the frequency range. At the receiver the received signals are sent to fast Fourier transform (FFT) block, demodulator and parallel to serial converter where signals are converted back into their original form.

\section{ADAPTIVE MODULATIONS}

The benefits of OFDM are that each sub-channel is considerably narrowband and is assumed to have flat-fading. However, it is entirely possible that a certain number of sub-channel experience deep fading, resulting in a large BER. Thus, it is necessary to take advantage of sub-channels having relatively superior performance; this is the motivation 
for adaptive modulation. Adaptive modulation is an important method that provides better performance over non-adaptive non-coded schemes. An inherent assumption in adaptive loading technique is some form of channel knowledge at both the transmitter and receiver. The variation in fading statistic among subcarriers in some OFDM channels suggests that some good subcarriers with high instantaneous signal to noise ratio can be made to carry more bits and be allocated with less transmission power and vice versa for the weak subcarriers.

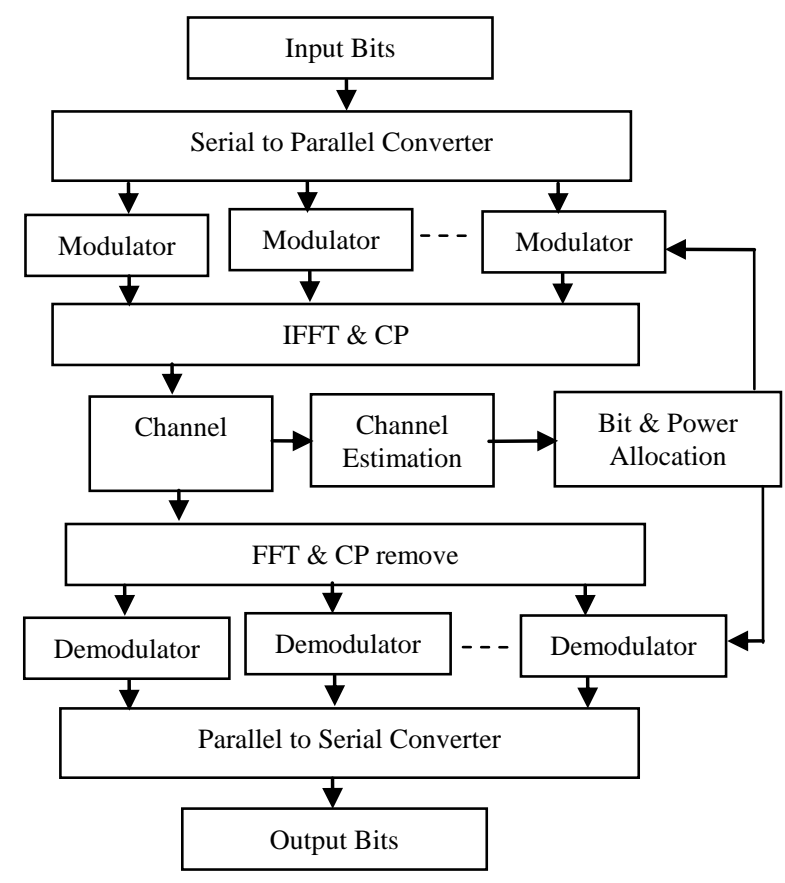

Fig. 1. OFDM system block structure

The optimal adaptive transmission methodology, which achieves the Shannon capacity for a fixed transmits power, is the water filling distribution of power over the frequency selective channel. However, while the water filling distribution will indeed gives the optimal solution, it is complex to compute, and it tacitly assumes infinite granularity in the constellation size, which is not practically realizable [5], [6].

\section{ADAPTIVE LOADING PRINCIPLES}

There are two types of link adaptation algorithms- those that try to improve data rate and those that try to enhance performance at a given fixed data rate [7].

Rate adaptive loading criterion: A rate- adaptive loading procedure increase the number of bits per symbols subject to fixed energy constraint:

$$
\max b=\sum_{n=1}^{N} \frac{1}{2} \log _{2}\left(1+\frac{\varepsilon_{n} \times g_{n}}{\Gamma}\right)
$$

Subjected to: $\quad N \bar{\varepsilon}_{n}=\sum_{n=1}^{N} \varepsilon_{n}$
Margin-Adaptive Loading Criterion: A margin-adaptive loading procedure minimizes the energy subject to a fixed bits/symbol constraint:

$$
\min \varepsilon_{x}=\sum_{n=1}^{N} \varepsilon_{n}
$$

Subjected to: $b=\sum_{n=1}^{N} \frac{1}{2} \log _{2}\left(1+\frac{\varepsilon_{n} \times g_{n}}{\Gamma}\right)$

where $\Gamma$ (or GAP) is called SNR-gap, is diffined as a ratio of ideal SNR at which system can transmit at C bits/symbol over a practical SNR at which the system can transmit $\mathrm{R}$ bits/symbol. It quantify how well the practical system compares to an ideal modulation system.

These link adaptation algorithms, computs the number of bits and energy required to transmit bits for each subchannel in a parellel set of subchannels.

The link adaptation algorithm utilized in this paper is an efficient approach to achive rate and power optimization based on the assumption that instantaneous channel state information is known at both the transmitter and receiver. Here only six different M-array Phase Sift Keying (M-PSK) signal constellations are used.

There are several algorithms proposed for bit and power loading based on criterions expressed above, but in this paper link adaptation was carried out using two algorithms: Chow Algorithm and Campello Algorithm [8], [9]. To initialize the bit loading each sub channel was allocated energy utilizing Chow's Algorithm and at the same time it was allocated maximum number of bits allowable per sub channel. There after bit allocation was done by Campello Algorithm.

\section{A. The Chow's Algorithm}

1) Compute sub channel signal to noise ratios based on the formula [8]:

$$
S N R_{i}=\frac{{\text { subcarrier } \text { gain }^{2}}_{\text {noise } \times \text { gap }}}{\text { gat }}
$$

2) Compute the number of bits for the $i^{t h}$ Sub-channel based on the formula:

$$
\bar{b}_{i}=\log _{2}\left(1+\frac{S N R_{i}}{g a p}\right)
$$

3) Round the value of $\bar{b}_{i}$ down to $b_{i}$

4) Restricts $b_{i}$ to take values $0,1,2,4,6$ or 8 (corresponding to available modulation order)

5) Compute the energy for the $i^{\text {th }}$ sub channel based on the number of bits initially assigned to it using the formula:

$$
e_{i}\left(b_{i}\right)=\left(2^{b_{i}}-1\right) / \text { gap }_{i}
$$

6) Form a table of energy increments for each sub channel. For the ith sub channel

$$
\Delta e_{i}(b)=e_{i}(b)-e_{i}(b-1)=2^{b-1} / \operatorname{gap}_{i}
$$


where

$$
\text { gap }_{i}=\frac{S N R_{i}}{\operatorname{gap}}
$$

'gap' is a tuning parameter for $E_{b} / N_{0}$. Different values of gap give different $E_{b} / N_{0}$ ratio for a desire number of bits to transmit.

\section{A. The Compello's Algorithm}

The basic concept of link adaptive compello's algorithm is that each increment of additional bits to be transpose by multi-channel transmission system is placed on the sub-channel that would require the least energy for its transport. Compello formalized an iterative algorithm that will translate any bit distribution into an efficient bit distribution. Efficiency means that there is no movement of a bit from one sub-channel to another that will reduce the symbol energy. This algorithm is based on $\beta$-tightness algorithm which is given as follow:

Set

$$
b_{\text {total }}=\sum_{n=1}^{N} b_{n}
$$

While

$$
b_{\text {total }} \neq b_{\text {target }}
$$

If

$$
\begin{gathered}
b_{\text {total }}>b_{\text {targ } e t} \\
n \leftarrow \arg \left[\max _{1<i<N}\left\{e_{i}\left(b_{i}+\beta\right)\right\}\right] \\
b_{\text {total }} \leftarrow b_{\text {total }}-\beta \\
b_{n} \leftarrow b_{n}-\beta
\end{gathered}
$$

Else

$$
\begin{gathered}
b_{\text {total }}<b_{\text {target }} \\
m \leftarrow \arg \left[\min _{1<i<N}\left\{e_{i}\left(b_{i}+\beta\right)\right\}\right] \\
b_{n} \leftarrow b_{n}+\beta
\end{gathered}
$$

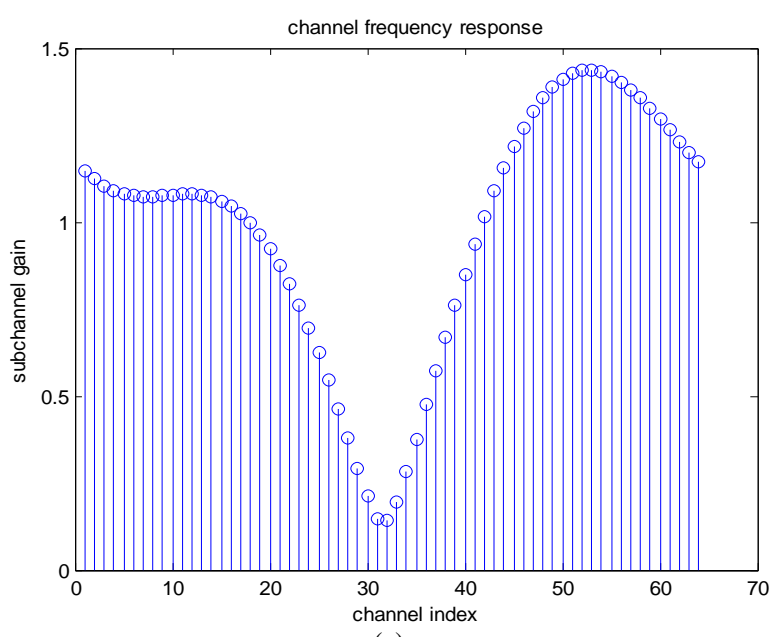

(a)

An example of implementing these two algorithms is indicated in Fig. 2. This figure indicates the typical channel frequency response, the discrete bit allocation to each subcarrier, and the corresponding energy on each subcarrier.
The sub-channel experiencing very deep fading had few or zero bits allocated to them. Also, note that the finite number of M-PSK constellations available means that the rate remains fixed over some intervals where the gain does not vary too widely.

\section{Simulations AND Results}

In this section optimal bit and power loading algorithm is simulated for frequency selective Rayleigh fading channel. The simulation results (BER v/s $\mathrm{E}_{\mathrm{b}} / \mathrm{N}_{0}$ performance analysis) for different coding schemes are presented in this section. In order to reduce the complexity of the system model, the entire system is considered as discrete time system.

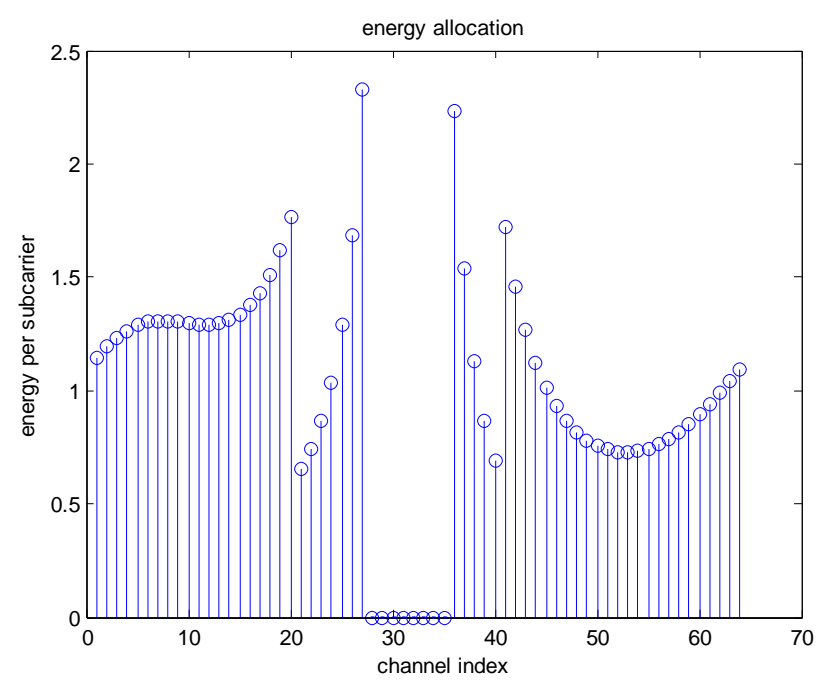

(b)

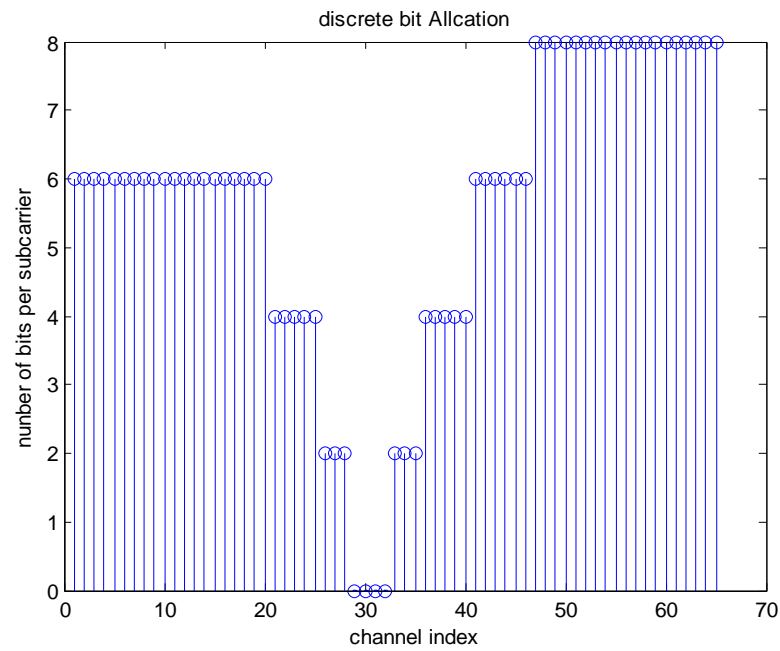

(c)

Fig. 2. An example of discrete bit and energy allocation

As the instantaneous channel state information is necessary at the both i.e., the transmitter and the receiver to implement the adaptive bit and power loading algorithms, perfect channel knowledge is assumed throughout the simulation. The configurations considered are for OFDM system with 64 subcarriers, 64 OFDM symbol time periods, and 16 symbol periods for guard time. The parameters were held constant throughout the simulation. M-PSK adaptive modulation for each subcarrier is used. The usable modulation modes are: 2PSK, 4PSK, 16 PSK, 64 PSK and 256 PSK. 
In Fig. 3, we provide the simulation result for Link Adaptive SISO-OFDM, for M-PSK, M-PSK with Gray coding and M-PSK with TCM. For all MIMO system $2 \times 2$ antenna configuration is considered. In Fig. 4 we provide simulation result for Link Adaptive MIMO-OFDM system.

\section{CONCLUSIONS}

Link adaptation algorithms with different coding schemes are investigated in this paper for SISO and MIMO-OFDM systems. We found that link adaptation algorithms improves the BER performance and minimizes the overall power input at the transmitter while maintaining a defined data rate constraint. With adaptive M-PSK, coding schemes like Gray code and TCM are introduced in the algorithm in this paper. These schemes give better BER performance over non coded algorithm.

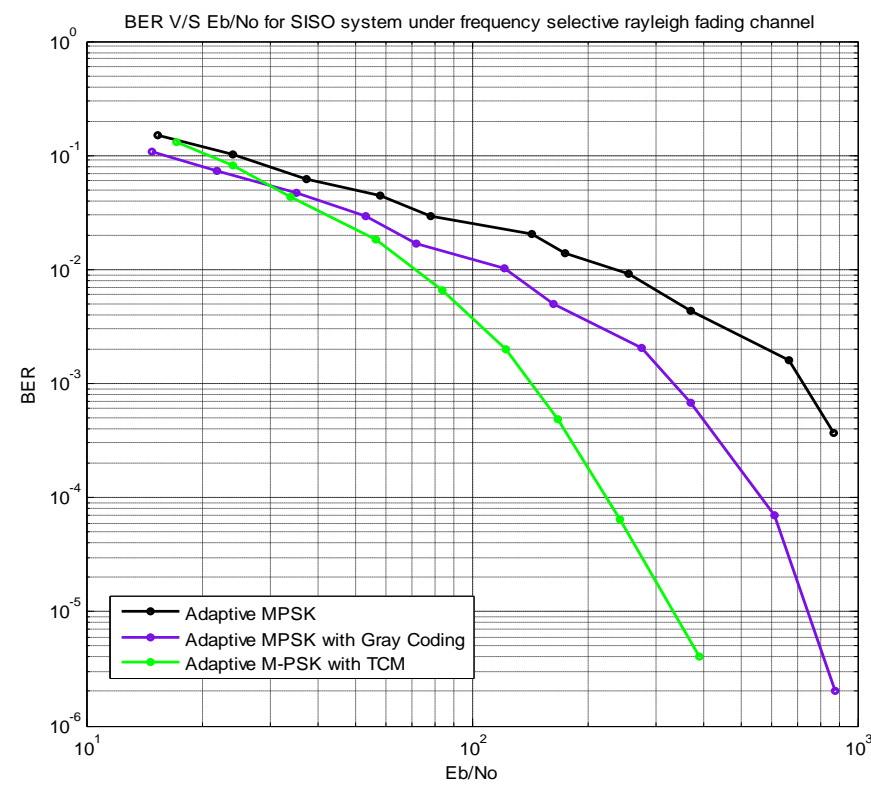

Fig. 3. Comparison of Adaptive SISO-OFDM system for different coding schemes

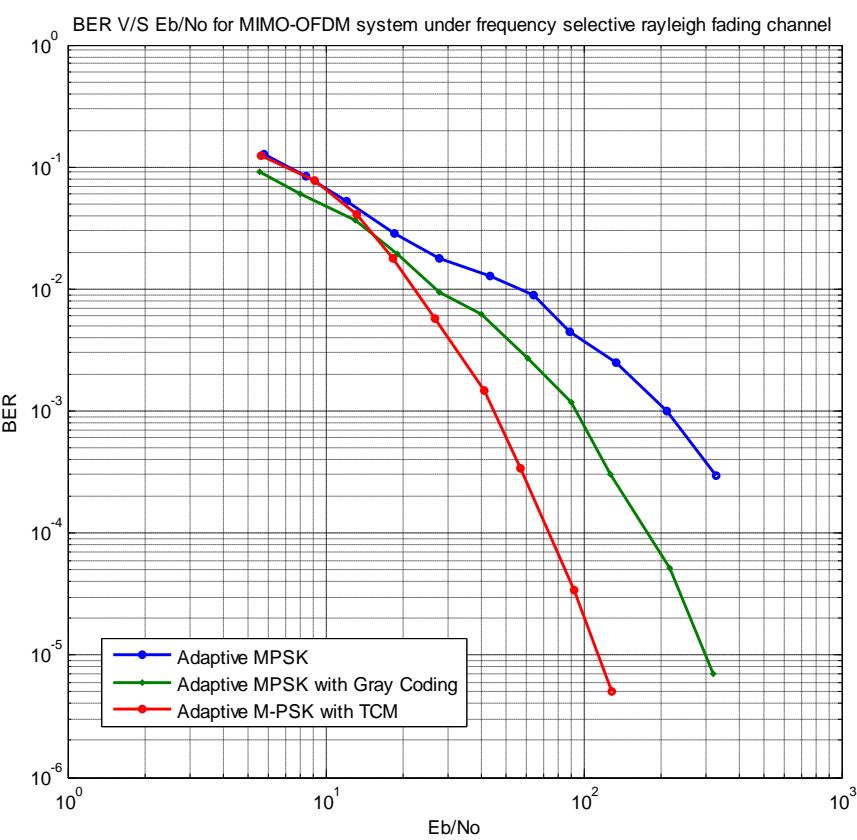

Fig. 4. Comparison of Adaptive MIMO-OFDM system for different coding schemes

\section{REFERENCES}

[1] R. Prashad, OFDM for wireless communication systems, Artech house publishes, London, 2004, ch. 1, pp. 1-18

[2] Y. Li and G. L. Stuber, Orthogonal Frequency Division Multiplexing for Wireless Communication, Springer Publication, U.S.A. 2006, ch. 2, pp. $19-46$

[3] L. Ben, S. Junde, S. Mei, and Z. Wenan, "Link Adaptation of Wideband OFDM Systems in Multipath Fading Channel," IEEE Canadian Conference on Electrical and Computer Engineering, 2002

[4] S. T. Chung and A. Goldsmith, "Degree of Freedom In Adaptive Modulation: A Unified View," IEEE Transactions on Communication, VTC'01, pp. 1267-1271, 2001

[5] T. Keller and L. Hanzo, "Adaptive modulation techniques for duplex transmission," IEEE Transcations on Vehicular Technology, vol. 49, no. 5, pp. 1893-1906, Sep. 2000

[6] J. Faezath and K. Sabria, "Adaptive Modulation for OFDM systems," International Journal of Communication Networks and Information Security (IJCNI), vol. 1, no. 2, pp. 1-8, August 2009

[7] L. Jiancai, Y. Wanwan, Z. Weili, W. Yafu, Y. Zhihui, X. Jiakai, and N Xinbao, "Adaptive bit and power loading algorithm with low complexity in MIMO-OFDM systems," IEEE international conference, 2009

[8] P. Chow, J. M. Cioffi, and J. A. C. Bingham, "A Practical Discrete Multitone Transceiver Loading Algorithm for Data Transmission Over Spectrally Shaped Channels," IEEE Trans. Comm., vol. 43, no. 2, pp. 773-775, February 1995

[9] J. Campello, "Practical bit loading for discrete multicarrier modulation systems," IEEE International Conference on Communications ICC99, pp. 801-805, 1999.

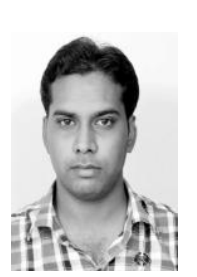

Sandeep Singh was born in Haridwar, India on 20/06/1986. He received his B.E degree in electronics and communication engineering from Kumaon Engineering College Dwarhat, India in the year 2008 He did his M.Tech in electronics and communication engineering from National Institute of Technology Jalandhar, India in the year 2012. His research interests include orthogonal frequency division multiplexing technique and cognitive radio technology.

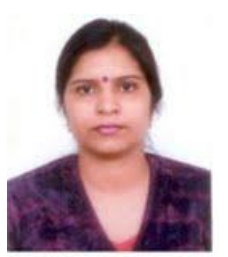

N. Sood received the B.Tech. degree (with Honour) in Electronics and Communication from Sant Longowa Institute of Engineering and Technology, India 2000. In year Jan. 2002, she completed her M.Eng. Degree (With Honour) in Electronics and Communication from Thapar University, Patiala, India. From year 2002 to 2007, she worked with DAV institute of Engineering and Technology, Jalandhar and Guru Nanak Dev University (Regional Campus), Jalandhar.Currently, she is working as Assistant Professor at Dr. B R Ambedkar National Institute of Technology, Jalanadhar and working towards her $\mathrm{PhD}$ degree. Her current research includes the simulation of wireless systems based on OFDM and simulation of fading channels. 